\title{
Distributed learning enhances relational memory consolidation
}

\author{
Leib Litman ${ }^{1}$ and Lila Davachi ${ }^{1-3}$ \\ ${ }^{1}$ Department of Psychology, New York University, New York, New York 10003, USA; ${ }^{2}$ Center for Neural Science, New York \\ University, New York, New York 10003,USA
}

\begin{abstract}
It has long been known that distributed learning (DL) provides a mnemonic advantage over massed learning (ML). However, the underlying mechanisms that drive this robust mnemonic effect remain largely unknown. In two experiments, we show that DL across a $24 \mathrm{hr}$ interval does not enhance immediate memory performance but instead slows the rate of forgetting relative to ML. Furthermore, we demonstrate that this savings in forgetting is specific to relational, but not item, memory. In the context of extant theories and knowledge of memory consolidation, these results suggest that an important mechanism underlying the mnemonic benefit of DL is enhanced memory consolidation. We speculate that synaptic strengthening mechanisms supporting long-term memory consolidation may be differentially mediated by the spacing of memory reactivation. These findings have broad implications for the scientific study of episodic memory consolidation and, more generally, for educational curriculum development and policy.
\end{abstract}

Over 100 years ago Jost put forth what is now known as his first law: "If two memory traces are of equal strength but different ages, the older one will benefit more from an additional learning trial." This general phenomenon, also known as the distributed learning effect (DL), refers to the finding that memory for items encoded over repeated sessions is better when those sessions are distributed across time rather than massed (ML). While hundreds of studies have now confirmed that DL provides a mnemonic advantage over ML (see Dempster 1988; Janiszewski et al. 2003; Cepeda et al. 2006 for reviews), the cognitive and neural mechanisms behind this effect remain unclear.

In this paper we present evidence supporting a consolidation theory account of DL. Consolidation is a neurobiological process involved in long-term stabilization of mnemonic representations, causing memories to strengthen and become less prone to forgetting and interference over time (e.g., Squire and Alvarez 1998; Dudai 2004; Wixted 2004). Historically, the field of psychology has been resistant to embracing consolidation as an explanation for DL memory enhancement. For example, in an influential symposium that captured the zeitgeist, Melton (1970) commented that "I find it comforting that no one in the symposium appealed to consolidation as an explanation of the observed distributed practice effects, but all sought instead to find understanding from hypotheses about the informationprocessing activities of the subject."

Instead of proposing neural mechanisms, researchers have favored accounts of DL effects in terms of cognitive factors, such as fatigue and encoding variability (Youtz 1941; Melton 1970; Hintzman 1974). Importantly, however, these theories have been derived from a DL literature that has primarily used very short intersession intervals (ISIs) ranging only from seconds to minutes (Underwood 1961; Melton 1970; Hintzman 1974) that are not likely to be affected by consolidation processes. For example, protein synthesis takes place over many hours (Tully et al. 1994) and systems-level consolidation continues for days and months (Squire and Alvarez 1998; Dudai 2004). The use of short ISIs may

\footnotetext{
${ }^{3}$ Corresponding author.
}

E-mail lila.davachi@nyu.edu; fax (212) 995-4349.

Article is online at http://www.learnmem.org/cgi/doi/10.1101/lm.1132008. at least partly explain why consolidation theory has played such a minor role in theories of DL.

These theoretical frameworks, which minimized the role of consolidation in DL, had the unfortunate consequence of keeping the field from realizing a promising behavioral paradigm for studying episodic memory consolidation. As a result, it is surprising to note that despite the great role that consolidation plays in the neuroscientific literature of memory, it is virtually not studied at all in the behavioral psychological literature. This is, at least in part, because few behavioral paradigms exist for investigating consolidation in neurologically intact subjects.

Here, we draw on both behavioral and neuroscience data to reintroduce consolidation theory as a plausible account of at least some forms of DL thereby bolstering DL as a means to studying memory consolidation. Our specific aim is to test two critical predictions. The first is based on the fact that consolidation slows the rate of forgetting (Wixted 2004) leading to the prediction that the DL advantage over ML should be specific to the rate at which memories are forgotten and not necessarily the overall probability of remembering at any one time point. The second prediction is informed by theories of memory consolidation, extant neuroimaging, and neuropsychological evidence suggesting (1) that the hippocampus is differentially important for relational, as compared to item memory (Davachi et al. 2003, 2006; Ranganath et al. 2004; Staresina and Davachi 2006; Mayes et al. 2007), ${ }^{4}$ and (2) that memory retrieval is initially hippocampal dependent and that over time this dependence wanes such that successful retrieval can be achieved without an intact hippocampus (Squire and Alvarez 1998; Dudai 2004). It follows from this account that DL should slow the rate of forgetting more so for relational as compared to item memory.

We tested these predictions in two behavioral studies. In each study we asked whether DL differentially slows the rate of relational forgetting over a $24 \mathrm{hr}$ time interval as compared to ML. In order to assess forgetting rate, memory must be assessed at least twice. Thus, memory was assessed both immediately after the second encoding session and then again $24 \mathrm{hr}$ later, allowing

${ }^{4}$ Relational memory can be defined as memory for the contextual or associative aspects of a prior experience (Cohen and Eichenbaum 1993). 
us to examine whether DL affects the strength of the initial memory trace independent of the rate of forgetting (Fig. 1).

Both experiments show that DL and ML do not differ with regard to the initial memory trace but that DL slows the rate of forgetting from the immediate to the delayed test. Critically, we also demonstrate that slowed forgetting after DL is specific to relational memory and does not appear to impact item recognition, at least in the present paradigm. These data support the idea that DL is, at least in part, supported by an enhancement of hippocampally mediated memory consolidation.

\section{Results}

\section{Experiment 1}

\section{Results}

To assess relational memory, the percent correct INTACT "intact" (II) pairs were compared across three conditions (Fig. 2A). The responses from the DL, ML, and the single session (SS) conditions on both tests were analyzed using a test (Test 1 , Test 2$) \times$ condition (DL, ML, SS) repeated-measures ANOVA which revealed a significant main effect of condition, $F_{(2,8)}=46, P<0.05$, test, $F_{(1,9)}=17, P<0.05$, and a significant condition $\times$ test interaction, $F_{(2,8)}=4.7, P<0.05$. Follow-up planned contrasts were then performed to further examine the condition differences. Performance on Test 1 did not differ between DL and ML word pairs, $t(9)=0, P=1$, while performance for SS word pairs was significantly worse than both DL, $t(9)=5.1, P<0.05$ and $\mathrm{ML}, t(9)=5.1$, $P<0.05$ conditions.

Twenty-four hours later, on Test 2, significant differences between all three conditions were observed. DL led to better memory performance than $\mathrm{ML}, t(9)=2.9, P<0.05$, and performance was better in the ML than the SS condition, $t(9)=12.2$, $P<0.05$. These results demonstrate that while DL does not enhance relational memory on an immediate memory test, it does enhance relational memory $24 \mathrm{hr}$ later.

A condition $(\mathrm{DL}, \mathrm{ML}) \times$ test (Test 1 , Test 2$)$ within-subjects ANOVA revealed a significant interaction, $F_{(1,9)}=8, P<0.05$, establishing that the rate of forgetting for DL word pairs was significantly slower than for ML pairs. Importantly, a condition $(\mathrm{ML}, \mathrm{SS}) \times$ test (Test 1 , Test 2 ) within-subjects ANOVA did not reveal this interaction between the ML and SS conditions, $F_{(1,9)}=0, P=0.98$. Thus, while memory performance in the ML condition was significantly better than in the SS condition on both the first and second tests, the magnitude of the ML advantage did not differ from Test 1 to Test 2, suggesting that memories were forgotten at a similar rate in both of these conditions. By contrast, DL did not provide a short-term advantage but did slow the rate of forgetting relative to ML.

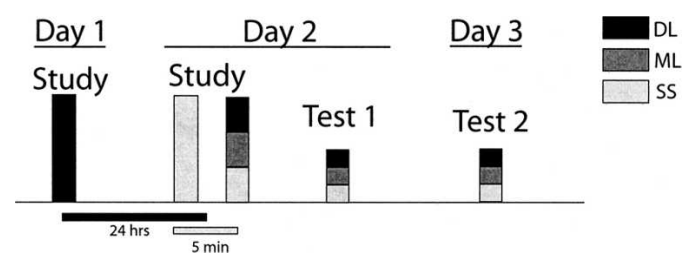

Figure 1. Study design: On Day 1 , subjects encoded the DL word pairs for the first time. On Day 2, during Phase 1 (light gray bar) subjects encoded the ML word pairs. During Phase 2 (beginning approximately 5 min after completion of Phase 1), all word pairs from the DL, ML, and SS conditions were (re)encoded in randomized order. After a short break, subjects were administered an associative recognition memory test for half of the stimuli from each of the three conditions. They returned $24 \mathrm{hr}$ later on Day 3 for a second retrieval session during which memory for the other half of the stimuli was tested.
A

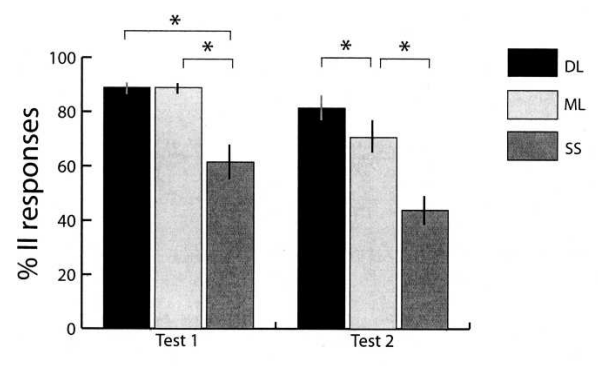

$\mathbf{B}$

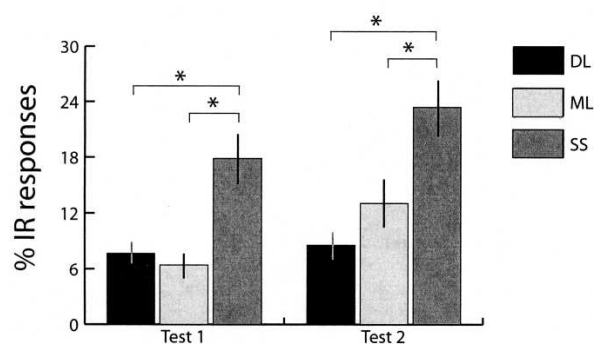

Figure 2. Results from Experiment 1. (A) Percentage of INTACT word pairs that were correctly identified as "intact" (\% II) on Test 1 and Test 2 for the DL, ML, and SS conditions. On Test 1 the INTACT SS word pairs were less likely to be identified as "intact" than both the DL and ML word pairs, while the $D L$ and $M L$ word pairs did not differ from each other. On Test 2 the INTACT DL word pairs were more likely to be identified as "intact" than ML word pairs that, in turn, were more likely to be identified as "intact" than the SS word pairs. (B) The percentage of INTACT word pairs that were incorrectly identified as "rearranged" (\% IR) on Test 1 and Test 2 for the DL, ML, and SS conditions. On Test 1, INTACT word pairs in the SS condition were more likely to be identified as "rearranged" than word pairs in the ML conditions, and marginally more likely to be identified as "rearranged" than the DL word pairs. The INTACT DL and ML word pairs did not differ from each other in their likelihood of being identified as "rearranged." On Test 2, INTACT ML word pairs were more likely to be identified as "rearranged" than DL word pairs. INTACT SS word pairs were more likely to be identified as "rearranged" than both DL and ML word pairs.

We next looked at the INTACT "rearranged" (IR) responses in order to examine item memory outcome (Fig. 2B). Item memory outcome did not differ on the first $t(9)=0.8, P>0.05$ or the second test between DL and ML. In fact, on Test 2 the opposite pattern of results was observed in that there was a marginal ML advantage over DL $t(9)=0.22, P=0.052$.

Because the raw proportion of IR responses may not be an appropriate measure of item memory since the number of II responses puts an upper limit on the number of IR responses, we further examined item memory performance using an approach similar to the Independent K procedure (Yonelinas et al. 1998). Namely, we used a measure of item memory that is independent of II responses (see Jacoby et al. 1997; Yonelinas et al. 1998). In this procedure, which we will refer to as IRel to stand for "Independent of Relational memory," the proportion of IR responses is divided by the proportion of INTACT pairs that were not labeled "intact," IRel $=\% \mathrm{IR} /(100-\% \mathrm{II})$.

On Test 1, no IRel differences were observed between DL $($ IRel $=78$ ) and ML (IRel = 65), $t(15)=0.003, P>0.5$ but the IRel statistic for ML was significantly higher than SS (IRel $=48$ ), $t(15)=2.4, P>0.05$, while DL was marginally higher than SS, $t(15)=1.8, P=0.09$. On Test 2 , both DL (48) and ML (44) differed from SS (31) [DL, (15) $t=4.5, P<0.05$; ML, (15) = 3.7, $P<0.05$ ]. However, on Test 2, IRel for DL did not differ from ML, $t(9)=1.3$, 
$P>0.05$. Thus, using an independent measure of item memory, the results further support the conclusion that DL selectively impacts upon relational but not item memory.

\section{Experiment 2}

In Experiment 1, we found that DL enhances retention of relational memory. However, one potential difficulty with this interpretation is the possibility of a ceiling effect. First, it is possible that DL does provide an initial encoding advantage over ML but that performance in the two conditions is at ceiling as evidenced by the above $85 \%$ correct performance for both DL and ML word pairs. Also, the high number of II responses limits the number of responses available for the IRel analysis, thus limiting its sensitivity. Hence, in the present study, we examine how DL impacts upon both item and relational memory using cued-recall instead of associative recognition.

All word pairs whose paired associate was correctly recalled in this experiment are a subset of the trials on which subjects had correct item recognition. Thus, when subjects correctly recognize a word as being "old" they may or may not recollect additional relational detail. We therefore separated the recognized words into those whose paired associate was recalled and those whose paired-associate was not recalled, to yield the following three trial types: (1) $[\mathrm{R}+]$ a word was both correctly recognized and its paired associate was recalled (i.e., intact item recognition + intact relational memory); (2) [R - ] a word was correctly recognized but its paired associate was not recalled (i.e., intact item recognition with no measurable relational memory); and (3) [M] a word was forgotten (no item memory). The $\mathrm{R}+$ and $\mathrm{R}-$ trials are analyzed separately below.

We replicated the results of Experiment 1 (Fig. 3). Specifically, no differences were observed between DL and ML on Test 1 for $\mathrm{R}+$ trials (Fig. 3A), $t(15)=-1.6, P>0.05$ nor for $\mathrm{R}-$ trials (Fig. 3B), $t(15)=-1.6, P>0.05$. On Test $2, \mathrm{R}+$ performance for DL was better than ML (Fig. 3A), $t(15)=2.4, P<0.05$. However, unlike $\mathrm{R}+$ performance, $\mathrm{R}-$ performance did not differ between DL and ML on Test 2 (Fig. 3B) $t(15)=-0.3, P>0.05$. As expected $\mathrm{R}+$ performance for the SS condition was significantly worse than for the DL and ML conditions on both Test 1 and Test 2 [Test 1 $\mathrm{DL}, t(15)=6, P<0.05$, ML, $t(15)=-2.5, P<0.05$; Test 2 , and DL, $t(12)=5.9, P>0.05 \mathrm{ML}, t(12)=5.9, P>0.05]$.

We additionally observed a significant test (Test 1 , Test $2) \times$ condition $(\mathrm{DL}$ and $\mathrm{ML}$ ) interaction for the $\mathrm{R}+$ responses $\left(F_{(1,15)}=8.2, P<0.05\right)$ but not for the $\mathrm{R}-$ responses $\left(F_{(1,15)}=0.24, P>0.6\right)$ replicating the finding that relational memory, but not item recognition, decay is slower following DL compared to ML.

We then examined item memory performance using the IRel procedure (Yonelinas et al. 1998). As in Experiment 1, in this procedure the proportion of trials for which subjects have intact item memory is divided by the proportion of responses where subjects do not have intact relational memory, $I R e l=\% R-1$ $(100-\% \mathrm{R}+)$. On Test 1 , no IRel differences were observed between DL (IRel = 59) and ML (IRel = 59), $t(15)=0.003, P>0.05$. IRel for ML was significantly higher than SS (IRel $=48$ ), $t(15)=2.4, P>0.05$, while DL was marginally higher than SS, $t(15)=1.8, P=0.09$. On Test 2 , IRel for DL (0.48) also did not differ from ML (IRel $=44), t(15)=1.3, P>0.05$ and both DL and ML differed from SS (IRel $=31$ ) [DL, (15) $t=4.5, P<0.05$; ML, (15) $=3.7, P<0.05]$. Thus, using IRel measures, the results further support the conclusion that DL selectively impacts upon relational but not item memory.

A potential theoretical problem with our analyses is that group differences may be driven by stable underling subject effects. Namely, individual subjects with DL advantages on both
A

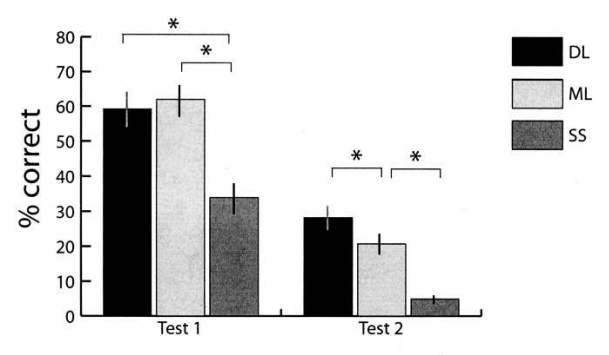

$\mathbf{B}$

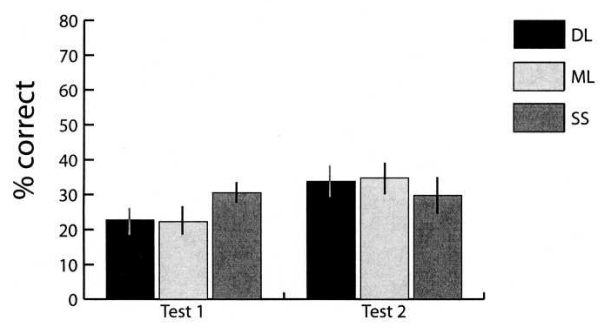

C

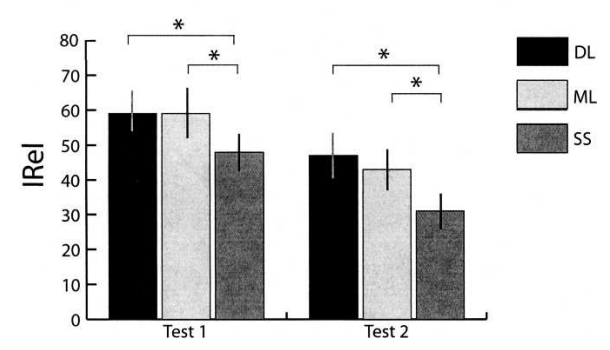

Figure 3. Results from Experiment 2. The figure shows $(A)$ cued recall, $(B)$ recognition without recollection, and $(C)$ item recognition independent of relational memory (IRel) performance of the $\mathrm{DL}, \mathrm{ML}$, and SS conditions across Test 1 and Test 2. (A) For Test 1 , on the cued-recall test, the SS word pairs were less likely to be recalled than both the DL and ML word pairs. Performance for the $\mathrm{DL}$ and $\mathrm{ML}$ word pairs did not differ. On Test 2 cued-recall for DL word pairs was better than for ML word pairs and ML word pair performance was better than that seen for the SS word pairs. (B) On Test 1, more SS words were recognized without recall of the paired associate (item recognition only) than both the $\mathrm{DL}$ and $\mathrm{ML}$ words. $\mathrm{DL}$ and $\mathrm{ML}$ item recognition did not differ from each other. On Test 2, no significant condition differences were seen. (C) To further characterize item memory performance, we employed an analog of the independent $\mathrm{K}$ procedure (I Rel). This ratio measured the percentage of items that were recognized without recollection from all nonrecollected word pairs $[R-/$ $(100-\mathrm{R}+)]$. Across both tests, no differences were found between DL and ML while SS item memory was lower than both.

Test 1 and Test 2 may be mixed with subjects showing ML advantages. To alleviate the concern that the lack of a difference in memory performance on Test 1 results from group averaging, we examined the performance of only the subjects who showed a numerical DL advantage on Test 2 ( $N$ 's $=8 / 10$ and 12/16 for Experiments $1 / 2$, respectively) to see if any hint of a Test 1 advantage would then emerge (Fig. 4). Across both experiments, no differences on Test 1 between the DL and ML conditions were evident (all $P^{\prime}$ s $>0.05$ ) even for only the subjects with a DL advantage on Test 2 . 
A

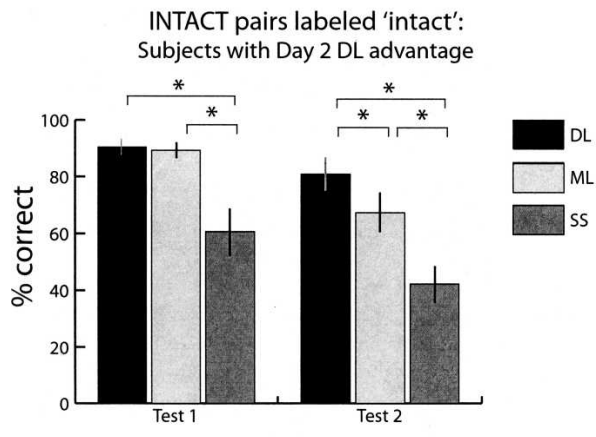

B

Cued recall: Subjects with Day $2 \mathrm{DL}$ advantage

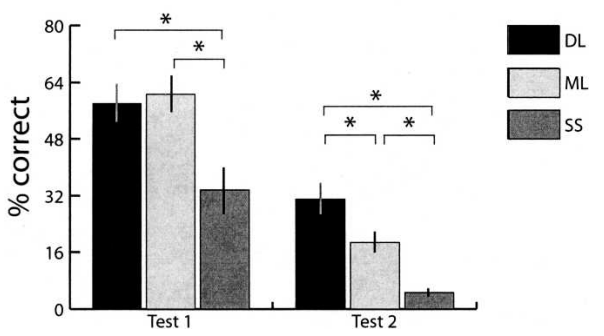

Figure 4. Results from Experiments 1 and 2 reanalyzed including only the subjects whose numerical DL performance on Test 2 was better than ML. Across both experiments no differences on Test 1 were observed between $\mathrm{DL}$ and ML conditions for these subjects.

\section{Discussion}

We operationally defined consolidation as a time-related enhancement of mnemonic representations that reveals itself behaviorally through a decreased rate of forgetting. We hypothesized, based on previous theoretical work (Wixted 2004), that a consolidated memory is one that is less likely to be forgotten than a memory that has not undergone a process of consolidation. In two experiments, we showed that distributing learning over a $24 \mathrm{hr}$ interval slowed the rate of forgetting without having a measurable effect on the initial memory trace. Critically this pattern of results was specific for relational, but not item, memory. To our knowledge, these results are the first to systematically demonstrate that DL leads to less forgetting, or enhanced consolidation, of relational memories while leaving item recognition broadly unchanged. These results suggest that DL specifically enhances hippocampally mediated memory consolidation.

We interpret these results in light of consolidation theory. The possibility that DL enhances consolidation provides an account of DL that is well grounded in contemporary knowledge of both cellular and systems level brain processes. On a cellular level, these findings may indicate that synaptic strengthening mechanisms supporting consolidation (Hasselmo et al. 1996) may be differentially mediated by the spacing of memory reactivation. This view is consistent with the animal literature where it is widely accepted that a time interval between encoding sessions is necessary for protein synthesis to support long-term memory consolidation (Carew and Kandel 1973; Tully et al. 1994; Menzel 2001; Liu et al. 2008), and with what is known about the impact of sleep on memory consolidation (Walker and Stickgold 2006).

These findings also have strong implications for systemlevel accounts of consolidation that propose a hippocampalneocortical interplay as being critical for the stabilization of memory over time. As mentioned, the hippocampus has been consistently shown to support the relational binding of items within an event (Davachi et al. 2003; Ranganath et al. 2004; Davachi 2006; Staresina and Davachi 2006; Mayes et al. 2007). Our behavioral data showing a relational memory benefit following the DL procedure underscore the notion that these relational links, likely supported by hippocampal mechanisms, may not be fully stabilized following a single encoding session. Rather, these links may begin to strengthen, or consolidate, over a $24 \mathrm{hr}$ interval. The present data suggest that reactivation following this initial period of consolidation, as compared to more immediate reactivation, renders these memories more resistant to forgetting. Consistent with our behavioral data, some initial studies of human memory consolidation using brain imaging report increased activation of the hippocampus following a $24 \mathrm{hr}$ retention interval as compared to intervals of less than an hour (Bosshardt et al. 2005; Litman and Davachi 2007; but see Stark and Squire 2000; Takashima et al. 2006;). Our data complement these imaging findings in revealing the behavioral consequences of consolidation, namely a reduced forgetting of relational information.

While we see a differential benefit for relational memory as compared to item memory consolidation in this particular paradigm, this is not to say that item memories do not undergo a process of consolidation at all. While most tasks that are used to examine memory consolidation in the animal literature, such as contextual fear conditioning and socially acquired food preference, have a strong relational component (for review, see Frankland and Bontempi 2005), these studies typically do not measure item memory separately from relational memory consolidation. Thus, currently, little is known about the differential time course and neural mechanisms of item and relational episodic memory consolidation. Nonetheless, some recent work in human procedural memory has reported similar dissociations between item and relational memory consolidation. Litman (2005) showed that relational motor memory requires a 24 -hr period of consolidation. On the other hand, when a motor task does not have a relational component, consolidation was observed within just a few hours. Likewise, a recent paper by Ellenbogen et al. (2007) found that relational memory measured using performance on the furthest pair of a transitive inference task benefits the most from sleep. Taken together, these studies and our own suggest that relational and item memory may not share the same time course of consolidation and may not be mediated by the same neural mechanisms. Future work will be needed to gain leverage on this question.

Previous examinations of the differential effects of DL at long- and short-term study-test intervals have been mixed. For example, while some studies show that DL has a long-term but not a short-term memory advantage, at least under some conditions of DL (see Cepeda et al. 2006), DL has a beneficial effect at both short and long retention intervals. If the long-term DL benefit were always accompanied by a short-term DL benefit, a memory consolidation account would be weakened substantially because the presence of an initial DL benefit would leave open the possibility that encoding under conditions of ML is inferior at the outset and that the long-term DL advantage is, at least in part, a product of inferior encoding during ML. The critical point made by the present studies is that the long-term relational DL benefit is evident even in the absence of an initial DL advantage, both on item and relational memory measures, and is therefore unlikely to be parsimoniously explained as the result of inferior encoding during ML.

Hence, the present pattern of results cannot be easily accommodated by either a fatigue or an encoding variability account because, like an inferior encoding explanation, it is not clear why fatigue and encoding variability should specifically slow forgetting rates and should not impact short-term test per- 
formance (however, see Cepeda et al. 2006). Furthermore, encoding variability and fatigue theory provide no principled account of why the DL benefit would be specific to relational but not item memory or why differential relational memory effects will be seen across Tests 1 and 2. Another possibility is that, under conditions of DL, a second study session establishes a second independent memory trace. Thus, a 24-hr interval between learning sessions may be more likely to result in distinct memory traces that are more likely to be available at retrieval since they will not be interfered with by other, overlapping traces. However, it is unclear how a multiple-trace explanation would predict our findings that DL did not result in enhanced memory on both memory tests but, instead, slowed the rate of memory decay. Nonetheless, these are important issues for consideration in future work.

Within the consolidation framework, several factors may interactively contribute to the DL benefit. The first, as already mentioned, is the time period of consolidation between distributed encoding sessions, which helps to stabilize mnemonic traces. The second factor is the reactivation of mnemonic representations during the second encoding session, which may further facilitate consolidation. It has been suggested that reactivating a memory trace after a period of consolidation initiates a process of reconsolidation (Dudai 2006) that provides an additional consolidation opportunity. Thus, the reactivation of a memory may be as important for enhancing long-term memory, as is a period of consolidation. Additionally, in the absence of reactivation, memory decay may obscure any benefit that consolidation has conferred.

Consistent with the view that reactivation plays an important role in consolidation-related post-encoding memory stabilization, Hupbach et al. (2008) recently reported that memory reactivation after $24 \mathrm{hr}$ leads to episodic memory reconsolidation. In their study, reactivation was found to be critical in order for novel information to become incorporated into an already established representation, thus showing that reactivation initiates a reconsolidation process that further transforms a memory trace. Our findings converge in showing that reactivation following a consolidation period leads to constructive post-encoding processes, ones that lead to memory enhancement and reorganization.

Finally, as has been noted previously on many occasions, (Dempster 1988; Cepeda et al. 2006) the DL effect has clear educational relevance as a manipulation that enhances the effectiveness of the study. In this context, our finding that the longterm benefits of DL are specific to relational memory is important because it suggests that DL may aid students in forming relational, rather than rote, knowledge where individual facts learned in the course of study cohere to form a more meaningful whole. Additionally, in light of the idea that memory reactivation is a critical factor influencing episodic memory consolidation, it is natural to ask what kind of other variables may additionally contribute to this effect. For example, repeated testing has been shown to be more beneficial for long-term memory than repeated study (Roediger and Karpicke 2006). Thus, future investigations should examine the impact of combining the DL procedure with manipulations such as repeated testing, especially in educationally relevant settings.

The findings of our study strongly suggest that a $24 \mathrm{hr}$ interval may slow the rate of relational memory decay by allowing initial memories to consolidate before their reactivation. It therefore appears that the examination of long ISIs, with a particular emphasis on the way in which these manipulations slow the rate of relational memory forgetting, may prove especially useful in future investigations of episodic memory consolidation. The general idea that a time period following encoding is necessary in order to stabilize an episodic memory trace is well established. However, few behavioral paradigms exist with which one is able to study the consolidation of episodic memory in neurologically intact human populations. Therefore, our findings suggest that although the DL effect has been known for a long time, it may now find new applications in studies of episodic memory consolidation.

\section{Methods}

\section{Experiment 1}

\section{Subjects}

Ten subjects (average age, 22) participated in Experiment 1 and were paid \$30US for their participation. Six of the subjects were female.

\section{General design}

Immediate and delayed associative recognition performance following three different study conditions was examined: DL, ML, and a condition with only a single encoding session (SS). A within-subjects design was used such that each subject participated in all encoding and testing conditions (see Fig. 1). One hundred sixty DL word pairs were encoded twice with a $24 \mathrm{hr}$ intersession interval (on Days 1 and 2). One hundred sixty ML word pairs were encoded twice on the same day, Day 2. 160 SS word pairs were encoded once on Day 2. The first presentation of the ML word pairs took place in a separate experimental session. Following this session, during the second Day 2 encoding session, subjects encoded the DL, ML, and SS word pairs in random order. Memory for half of the word pairs from all three conditions was tested following the second encoding session on Day 2 and, for the other half of the word pairs, $24 \mathrm{hr}$ later on Day 3.

\section{Encoding stimuli and task}

The stimuli used in both experiments were pairs of concrete nouns each ranging from three to 10 letters in length. During each encoding trial, a word pair was presented on a computer screen for 5 sec. During this time, subjects were instructed to construct a vivid mental image containing the referents of the words interacting, and they were also asked to rate the difficulty of the task for each pair on a scale of 1-3.

\section{Retrieval stimuli and task}

The retrieval tests were designed to allow specific assessment of relational memory outcome. Of the 160 word pairs in each condition, 100 were presented INTACT (i.e., both old words that were previously paired during encoding) and 60 were presented REARRANGED (both old words but were not paired together during encoding). In addition, 160 novel foils consisting of two novel words were presented. During retrieval, subjects were asked to indicate whether each presented word pair was (a) "intact," (b) "rearranged," (c) one word only was "new," or (d) both words were "new." This procedure provides a measure of relational memory, as the trials for which subjects recognize an INTACT word pair as "intact" (II) indicate that (a) they recognize each item, and, critically, (b) they remember that the items had been previously paired together (Giovanello et al. 2004; Jackson and Schacter 2004; Kirwan and Stark 2004). This procedure also provides a measure of item memory, as the trials for which subjects label an INTACT word pair as "rearranged" (IR) indicate that (a) they recognize each item but that (b) they do not remember that the items had been previously paired together.

\section{Experiment 2}

\section{Subjects}

Sixteen subjects (average age, 22) were recruited from the NYU subject pool and were given course credit for their participation. Ten of the subjects were female. 


\section{General design}

The general design of Experiment 2 was the same as Experiment 1 except cued recall was used rather than word pair recognition. Additionally, the number of word pairs in each condition was reduced to 50 with a total of 150 pairs across the three conditions. Thus, each of the two memory tests consisted of 75 old word pairs, 25 from each of the DL, ML, and SS conditions. In the retrieval phase, words were presented one at a time and subjects were first asked to make an old/new judgment. If they correctly identified a word as being "old," subjects were then asked to recall that word's paired associate by typing it on the keyboard. With this procedure, we were able to examine both relational memory outcome as well as intact item recognition in situations where subjects could not correctly recall the paired associate.

\section{Acknowledgments}

The authors thank Kristine Wilkens for assistance in data collection. Grant support was provided by Helicon Therapeutics to L.D.

\section{References}

Bosshardt, S., Schmidt, C.F., Jaermann, T., Degonda, N., Boesiger, P., Nitsch, R.M., Hock, C., and Henke, K. 2005. Effects of memory consolidation on human hippocampal activity during retrieval. Cortex 41: 486-498.

Carew, T.J. and Kandel, E.R. 1973. Acquisition and retention of long-term habituation in aplisia: Correlation of behavioral and cellular processes. Science 182: 1158-1160.

Cepeda, N.J., Pashler, H., Vul, E., Wixted, J.T., and Rohrer, D. 2006. Distributed practice in verbal recall tasks: A review and quantitative synthesis. Psychol. Bull. 132: 354-380.

Cohen, N.J. and Eichenbaum, H.E. 1993. Memory, amnesia, and the hippocampal system. MIT Press, Cambridge, MA.

Davachi, L. 2006. Item, context and relational episodic encoding in humans. Curr. Opin. Neurobiol. 16: 693-700.

Davachi, L., Mitchell, J.P., and Wagner, A.D. 2003. Multiple routes to memory: Distinct medial temporal lobe processes build item and source memories. Proc. Natl. Acad. Sci. 100: 2157-2162.

Dempster, F.N. 1988. The spacing effect. Am. Psychol. 43: 627-634.

Dudai, Y. 2004. The neurobiology of consolidations, or, how stable is the engram? Annu. Rev. Psychol. 55: 51-86.

Dudai, Y. 2006. Reconsolidation: The advantage of being refocused. Curr. Opin. Neurobiol. 16: 174-178.

Ellenbogen, J., Hu, P., Payne, J.D., Titone, D., and Walker, M.P. 2007. Human relational memory requires time and sleep. Proc. Natl. Acad. Sci. 104: 7723-7728.

Frankland, P.W. and Bontempi, B. 2005. The organization of recent and remote memories. Nat. Rev. Neurosci. 6: 119-130.

Giovanello, K.S., Schnyer, D.M., and Verfaellie, M. 2004. A critical role for the anterior hippocampus in relational memory: Evidence from an fMRI study comparing associative and item recognition. Hippocampus 14: 5-8.

Hasselmo, M.E., Wyble, B.P., and Wallenstein, G.V. 1996. Encoding and retrieval of episodic memories: Role of cholinergic and GABAergic modulation in the hippocampus. Hippocampus 6: 693-708.

Hintzman, D.L. 1974. Theoretical implications of the spacing effect. Lawrence Erlbaum, Mahwah, NJ.

Hupbach, A., Gomez, R., Hardt, O., and Nadel, L. 2008. Reconsolidation of episodic memories: A subtle reminder triggers integration of new information. Learn. Mem. 14: 47-53.

Jackson, O. and Schacter, D.L. 2004. Encoding activity in anterior medial temporal lobe supports subsequent associative recognition. Neuroimage 21: 456-462.

Jacoby, L.L., Yonelinas, A.P., and Jennings, J.M. 1997. The relation between conscious and unconscious (automatic) influences: A declaration of independence. In Scientific approaches to consciousness (eds. J.D. Cohen and J.W. Schooler), pp. 13-47. Lawrence Erlbaum, Mahwah, NJ.

Janiszewski, C., Noel, H., and Sawyer, A.G. 2003. A meta-analysis of the spacing effect in verbal learning: Implications for research on advertising repetition and consumer memory. J. Consum. Res. 30: $138-149$.

Kirwan, C.B. and Stark, C.E.L. 2004. Medial temporal lobe activation during encoding and retrieval of novel face-name pairs. Hippocampus 14: $919-930$.

Litman, L. 2005. "Consolidation of procedural knowledge.” Ph.D. thesis, City University of New York.

Litman, L. and Davachi, L. 2007. When less means more: Hippocampal repetition suppression is correlated with the stability of relational memory. In Proceedings of the 37th Annual Meeting of the Society for Neuroscience San Diego, CA.

Liu, R.-Y., Fioravante, D., and Byrne, J.H. 2008. cAMP response element-binding protein 1 feedback loop is necessary for consolidation of long-term synaptic facilitation in Aplysia. J. Neurosci. 28: 1970-1976.

Mayes, A., Montaldi, D., and Migo, E. 2007. Associative memory and the medial temporal lobes. Trends Cogn. Sci. 11: 126-135.

Melton, A.W. 1970. The situation with respect to the spacing of repetitions and memory. J. Verb. Learn. Verb. Behav. 9: 596-606.

Menzel, R. 2001. Massed and spaced learning in honeybees: The role of CS, US, the intertrial interval, and the test interval. Learn. Mem. 8: $198-208$.

Ranganath, C., Yonelinas, A.P., Cohen, M.X., Dy, C.J., Tom, S.M., and D'Esposito, M. 2004. Dissociable correlates of recollection and familiarity within the medial temporal lobes. Neuropsychologia 42: $2-13$.

Roediger, H.L. and Karpicke, J.D. 2006. Test-enhanced learning: Taking memory tests improves long-term retention. Psychol. Sci. 17: 249-255.

Squire, L.R. and Alvarez, P. 1998. Retrograde amnesia and memory consolidation: A neurobiological perspective. Curr. Opin.Cogn. Neurosci. 5: 169-177.

Staresina, B.P. and Davachi, L. 2006. Differential encoding mechanisms for subsequent associative recognition and free recall. J. Neurosci. 26: 9162-9172.

Stark, C.E.L. and Squire, L.R. 2000. fMRI activity in the medial temporal lobe during recognition memory as a function of study-test interval. Hippocampus 10: $329-337$.

Takashima, A., Petersson, K.M., Rutters, F., Tendolkar, I., Jensen, O., Zwarts, M.J., McNaughton, B.L., and Fernandez, G. 2006. Declarative memory consolidation in humans: A prospective functional magnetic resonance imaging study. Proc. Natl. Acad. Sci. 103: 756-761.

Tully, T., Preat, T., Boynton, S.C., and Del Vecchio, M. 1994. Genetic dissection of consolidated memory in Drosophila. Cell 79: 35-47.

Underwood, B.J. 1961. Ten years of massed practice on distributed practice. Psychol. Rev. 68: 229-247.

Walker, M.P. and Stickgold, R. 2006. Sleep, memory and plasticity. Ann. Rev. Psychol. 57: 139-166.

Wixted, J.T. 2004. The psychology and neuroscience of forgetting. Ann. Rev. Psychol. 55: 235-269.

Yonelinas, A.P., Kroll, E.A., Dobbins, I., Lazzara, M., and Knight, R.T. 1998. Recollection and familiarity deficits in amnesia: Convergence of remember/know, process dissociation, and receiver operating characteristic data. Neuropsychology 12: 323-339.

Youtz, A.C. 1941. An experimental evaluation of Jost's laws (psychological monographs). American Psychological Association, Washington, D.C.

Received June 30, 2008; accepted in revised form July 16, 2008. 


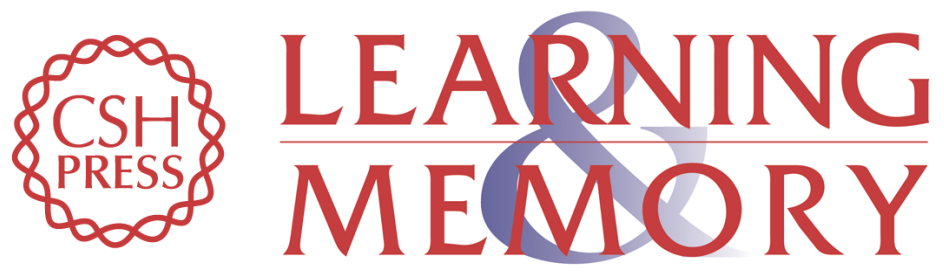

\section{Distributed learning enhances relational memory consolidation}

Leib Litman and Lila Davachi

Learn. Mem. 2008, 15:

Access the most recent version at doi:10.1101/lm.1132008

References This article cites 31 articles, 7 of which can be accessed free at: http://learnmem.cshlp.org/content/15/9/711.full.html\#ref-list-1

License

Email Alerting Receive free email alerts when new articles cite this article - sign up in the box at the Service top right corner of the article or click here. 